\title{
Brans-Dicke gravity and the capture of stars by black holes: some asymptotic results
}

\author{
Bertrand Chauvineau $^{1} \ddagger$, Alessandro D.A.M. Spallicci ${ }^{2} \S$ and \\ Jean-Daniel Fournier ${ }^{2} \|$ \\ UMR 6162 Dépt. d'Astrophysique Relativiste ARTEMIS \\ CNRS, Observatoire de la Côte d'Azur, France \\ 1 Avenue Copernic, 06130 Grasse \\ 2 Bd de l'Observatoire, 06304 Nice
}

\begin{abstract}
In the context of star capture by a black hole, a new noticeable difference between Brans-Dicke theory and general relativity gravitational radiation is pointed out. This feature stems from the non-stationarity of the black hole state, barring Hawking's theorem.
\end{abstract}

Emails : chauvineau@obs-azur.fr, spallicci@obs-nice.fr, fournier@obs-nice.fr

Pacs numbers : 04.50.+h, 04.30.Db

\section{Introduction}

General Relativity (GR) predicts radiation that, in the lowest order, is proportional to the third derivative of the quadrupole moment of the mass-energy distribution. It is a consequence of the conservation equations that the first derivative of the monopole moment and the second derivative of the dipole moment are zero. In Brans-Dicke (BD) theory (Brans and Dicke, 1961; Weinberg, 1972; Will, 1993) the scalar field $\Phi$ determines the local value of the gravitation "constant" G. A known feature of BD theory consists of the scalar field variability that generates contributions of dipolar terms to the gravitational radiation.

Herein, we show a new noticeable feature of BD theory in a non-stationary regime, which could happen after the capture of a star by a black hole. The BD vacuum field equations are

$$
\begin{aligned}
& R_{\alpha \beta}=\frac{1}{\Phi} \nabla_{\alpha} \partial_{\beta} \Phi+\frac{\omega}{\Phi^{2}} \partial_{\alpha} \Phi \partial_{\beta} \Phi, \\
& g^{\alpha \beta} \nabla_{\alpha} \partial_{\beta} \Phi=0,
\end{aligned}
$$

where $\Phi$ is the scalar field and $\omega$ its coupling to matter.

Since we are concerned in the post-capture phase, we write eq.(2) without source term. This does not imply that eq.(2) admits only trivial solutions; for instance, it allows solutions corresponding to a non vanishing gradient of the scalar field.

$\ddagger$ Member of CNAP, Comité National des Astronomes et Physiciens

$\S$ Associated member of CNRS, Centre National de la Recherche Scientifique

\| Member of CNRS, Centre National de la Recherche Scientifique 
Hawking's theorem (1972) on BD black holes, later generalised to the case of a scalar tensor black hole (Bekenstein and Meisels, 1978) states that under the following hypotheses:

(h1) : stationarity,

(h2) : asymptotical flatness,

(h3) : vacuum,

(h4) : existence of an event horizon $(H)$,

(h5) : finiteness of the area of $(H)$,

(h6) : bounded value of $\sqrt{|2 \omega+3|} \varphi \partial \varphi$ on $(H)$, where $\varphi=\Phi-\Phi_{0}\left(\Phi_{0}\right.$ being the asymptotic value of $\Phi$ at spatial infinity),

the solution of the BD field equations is a GR solution, i.e $\varphi \equiv 0$ everywhere; thus the common saying on BD black holes being identical to GR ones. We stress the importance of the concurrence of all the previous hypothesis, (h1-h6). Indeed, there are BD stationary black holes solutions which are not GR solutions due to the breakdown either of the hypothesis (h5) (Bronnikov et al., 1998) or (h2) (Singh and Rai, 1979; Kim, 1999).

Relaxing the hypothesis (h1), non-stationary BD black holes generally differ from the GR ones. In the present paper, we are interested in the problem of black hole relaxation after the capture of a star, in the framework of BD theory. As usual, we assume that the black hole under consideration satisfies the hypotheses (h2), (h5) and (h6). After the star has been captured, and during the relaxation phase, the hypothesis (h3) is assumed to be satisfied too. (h4) is naturally satisfied, since the endproduct is a black hole. Then, the above theorem ensures that the final (stationary) state is necessarily a GR black hole solution.

\section{The capture scenario in Brans-Dicke theory}

After the capture of a star, the black hole undergoes oscillations and relaxes towards a GR stationary state. Thus, adopting a perturbative approach, we rewrite the metric and the scalar field as

$$
\begin{aligned}
& g_{\alpha \beta}=\sigma_{\alpha \beta}+h_{\alpha \beta} \\
& \Phi=1+\varphi
\end{aligned}
$$

where $\sigma_{\alpha \beta}$ is a GR black hole metric (Schwarzschild or Kerr), and $\Phi_{0}=1$ is the normalized value of the scalar field at infinity, after relaxation. $\left|h_{\alpha \beta}\right|,\left|\partial h_{\alpha \beta}\right|,|\varphi|$ and $|\partial \varphi|$ are assumed to be small enough to ensure the applicability of the perturbative approach. Besides, $h_{\alpha \beta}, \varphi$ and their derivatives vanish in the large time limit, since the final state is the GR solution.

We assume that $\partial \varphi$ and $r_{B H} \partial \partial \varphi$ have the same order of magnitude, where $r_{B H}$ is a typical "length" associated to the black hole (for instance, the Schwarzschild radius, in the spherical case).

To lowest order, eq. (2) reads

$$
\partial_{\alpha}\left(\sqrt{-\sigma} \sigma^{\alpha \beta} \partial_{\beta} \varphi\right)=0 .
$$

Then, $\varphi$ decouples from $h_{\alpha \beta}$, and the integration of (5) gives, in principle, $\varphi$ from $\sigma_{\alpha \beta}$. In eq. (1), the Ricci tensor reads $R_{\alpha \beta}\left(g_{\mu \nu}\right)=R_{\alpha \beta}\left(\sigma_{\mu \nu}\right)+£_{\alpha \beta}\left(h_{\mu \nu}\right)=£_{\alpha \beta}\left(h_{\mu \nu}\right)$, since $\sigma_{\mu \nu}$ satisfies the GR vacuum equation $R_{\alpha \beta}=0$. The linear operator $£$ depends on the GR final state only, i.e. on its parameters (mass, angular momentum). 
An order of magnitude estimate of the r.h.s. terms of eq. (11) shows that $\Phi^{-1} \nabla_{\alpha} \partial_{\beta} \Phi \sim \bar{\nabla}_{\alpha} \partial_{\beta} \Phi \sim r_{B H}^{-1} \partial \varphi$, and $\omega \Phi^{-2} \partial_{\alpha} \Phi \partial_{\beta} \Phi \sim \omega \partial_{\alpha} \varphi \partial_{\beta} \varphi \sim \omega(\partial \varphi)^{2}$, where the operator $\bar{\nabla}$ is the covariant derivative with respect to the background metric $\sigma$. The term $\omega(\partial \varphi)^{2}$ is a second order term, but it is weighted by the BD parameter $\omega$, known to be very large. Then, even when $|\partial \varphi|$ is $\ll 1$ (but finite), the quadratic term can be comparable or greater than the linear one. Finally, eq. (11) reads

$$
£_{\alpha \beta}\left(h_{\mu \nu}\right)=S_{\alpha \beta},
$$

with

$$
\begin{aligned}
S_{\alpha \beta} & \simeq \omega \partial_{\alpha} \varphi \partial_{\beta} \varphi \text { when }|\partial \varphi|>\frac{1}{\omega r_{B H}} \quad \text { (quadratic phase) } \\
& \simeq \nabla_{\alpha} \partial_{\beta} \varphi \text { when }|\partial \varphi|<\frac{1}{\omega r_{B H}} \quad \text { (linear phase). }
\end{aligned}
$$

The quadratic phase occurs, or better may occur, just after the star absorption by the black hole. Conversely, the linear phase takes always place, before stationarity (unless the whole solution is a GR one, i.e. is such that $\Phi=$ constant). The quadratic phase doesn't necessarily occur. Indeed, solutions of eqs. (56) exist for which $\partial \varphi$ is always smaller than 1/ $\left(\omega r_{B H}\right)$ : an obvious example is provided by GR black hole oscillations, corresponding to the case $\varphi=0$ everywhere, a particular case of BD black hole oscillations.

But, if the initial values of $\partial \varphi$ are sufficiently large, such that the quadratic phase does take place, then the transition towards the linear phase does necessarily exist, due to the black hole acquisition of a GR stationary state at large times. At this stage, we can thus raise two questions :

(q1) : does such a transition, from the quadratic to the linear regime, in $\partial \varphi$, of the source, have a characteristic signature on $h_{\alpha \beta}$ ?

(q2) : is this signature essentially non dependent upon the precise form of the linear operator $£_{\alpha \beta}$ ?

\section{Discussion on the Schwarzschild case}

We consider the case where the final state is a Schwarzschild black hole. In this case, the scalar field equation (5) reads

$\frac{-r^{2}}{1-r_{g} / r} \frac{\partial^{2} \varphi}{\partial t^{2}}+\frac{\partial}{\partial r}\left[\left(r^{2}-r_{g} r\right) \frac{\partial \varphi}{\partial r}\right]+\frac{1}{\sin \theta} \frac{\partial}{\partial \theta}\left(\sin \theta \frac{\partial \varphi}{\partial \theta}\right)+\frac{1}{\sin ^{2} \theta} \frac{\partial^{2} \varphi}{\partial \phi^{2}}=0$,

where $r_{g}$ is the Schwarzschild radius.

The variables separation technique leads to the following particular solutions

$$
\varphi \equiv e^{-\lambda t} R(r) Y(\theta, \phi),
$$

where $\lambda$ is a positive separation constant and $Y(\theta, \phi)$ any spherical harmonic function. The radial function $R(r)$ has the following asymptotic forms

$$
\begin{aligned}
& R\left(r \simeq r_{g}\right) \simeq c_{1}\left(\frac{r}{r_{g}}-1\right)^{\lambda r_{g}} \\
& R(r \gg 1) \simeq c_{2} \frac{e^{-\lambda r}}{r}
\end{aligned}
$$


where $c_{1,2}$ are constants. It is noteworthy that the exponent in eq. (11) and the decrement in eq. (12) are expressed in terms of the constant $\lambda$, previously introduced as time decrement in eq. (10).

As consequence of the latter, the time-dependence of the source term in (6) is given by

$$
\begin{aligned}
S_{\alpha \beta} & \sim e^{-2 \lambda t} \quad \text { (quadratic phase) } \\
& \sim e^{-\lambda t} \quad \text { (linear phase). }
\end{aligned}
$$

The general solution of (6) reads then

$$
h_{\alpha \beta}=h_{\alpha \beta}^{(H)}+h_{\alpha \beta}^{(P)},
$$

where $h_{\alpha \beta}^{(H)}$ is the general solution of $£_{\alpha \beta}\left(h_{\mu \nu}\right)=0$ (GR case), and $h_{\alpha \beta}^{(P)}$ is a particular solution of the complete eq. (6). Since the linear operator $£_{\alpha \beta}$ is time-independent, $h_{\alpha \beta}^{(P)}$ can be constructed with the same time-dependence as $S_{\alpha \beta}$.

In those cases, where $h^{(P)}$ is not very small with respect to $h^{(H)}$, this will result into a change in the decrement of the signal, as direct consequence of the transition from the $e^{-2 \lambda t}$ to the $e^{-\lambda t}$ dependence in the source term. This decrement change, solely due to the presence of the BD scalar field, is to be perceived as a qualitative difference between BD and GR black hole oscillations.

\section{Conclusions}

We have identified a transition between two successive regimes during the radiation emission after capture of a star by a black hole. While such transition is obviously absent in general relativity, conversely it may be present in Brans-Dicke and concurrent scalar-tensor theories of gravity (Will, 1993).

\section{Acknowledgments}

The European Space Agency is acknowledged for granting the G. Colombo Senior Fellowship to A. Spallicci.

\section{References}

Bekenstein J D and Meisels A 1978 Phys. Rev. D 184378

Brans C and Dicke R H 1961 Phys Rev 124925

Bronnikov K A Clement G Constantinidis C P and Fabris J C 1998 Phys. Lett. A 243121

Hawking S W 1972 Commun. Math. Phys. 25167

Kim H 1999 Phys. Rev. D 60024001

Singh T Rai L N 1979 Gen. Rel. Grav. 1137

Weinberg S 1972 Gravitation and cosmology: principles and applications of the general theory of relativity John Wiley \& Sons

Will C M 1993 Theory and experiments in gravitational physics Cambridge Univ. Press 